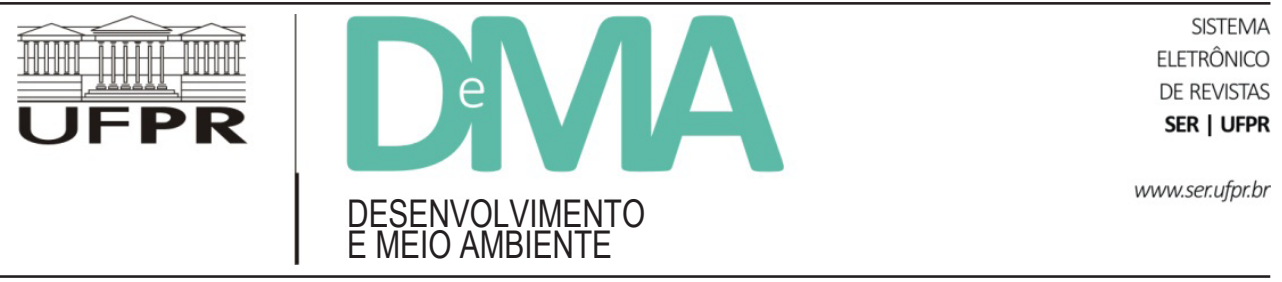

\title{
Gestión de playas urbanas: un análisis comparativo de los procesos de gobernanza en las playas Pocitos (Montevideo, Uruguay) y Central (Balneário Camboriú -SC, Brasil)
}

\author{
Gestão de praias urbanas: uma análise comparativa dos processos \\ de governança nas praias Pocitos (Montevidéu, Uruguai) e Central \\ (Balneário Camboriú - SC, Brasil)
}

\section{Urban Beach Management: A Comparative Analysis of Governance Processes in Pocitos (Montevideo, Uruguay) and Central (Balneário Camboriú - SC, Brazil) Beaches}

\author{
Briana BOMBANA ${ }^{1 *}$, Daniel CONDE ${ }^{2}$, Marcus POLETTE ${ }^{1}$ \\ ${ }^{1}$ Laboratório de Conservação e Gestão Costeira, Centro de Ciências Tecnológicas da Terra e do MAR (CTTMar), Universidade do Vale do \\ Itajaí (UNIVALI), Itajaí, SC, Brasil. \\ ${ }^{2}$ Centro Interdisciplinario de Manejo Costero Integrado (CURE), Universidad de la República (Udelar), Montevideo, Uruguay. \\ *E-mail de contacto: brianaab@gmail.com
}

Artículo recibido el 7 de noviembre, 2015, versión final aceptada el 7 de marzo, 2015.

RESUMEN: Tradicionalmente, la gestión de playas no ha fomentado la gobernanza, aspecto esencial para la sustentabilidad de estos ambientes costeros, dado que promueve acciones para el bien común. Este trabajo tiene el propósito de entender y comparar los procesos de gobernanza en la gestión de las playas Pocitos (Montevideo, Uruguay) y Central (Balneário Camboriú-SC, Brasil). Los objetivos propuestos fueron caracterizar el andamiaje institucional para la gestión de las playas en ambos casos, los stakeholders involucrados, y los resultados alcanzados, sugiriendo ajustes a los diseños y herramientas actuales. Entrevistas semi estructuradas fueron llevadas a cabo, y clasificadas en seis componentes de la gobernanza. La institución responsable por la gestión de playas y los demás stakeholders fueron abordados por medio de la técnica de la Bola de Nieve. En la playa de Pocitos, las actividades de gestión son realizadas a través de un proceso participativo e integrado, principalmente involucrando divisiones del gobierno, que conforman a un comité de trabajo semanal, que permite la implementación de los procesos de gobernanza. Sin embargo, son necesarias reformas, especialmente respecto a la participación de un amplio rango de stakeholders. En la playa Central, las actividades de gestión demuestran una débil integración entre las divisiones del gobierno municipal, y la participación de otros stakeholders no es usual. Una alternativa de gestión similar a la de Pocitos es sugerida para la playa Central. La contribución 
principal de este trabajo es un conjunto de recomendaciones para la gestión de ambas playas analizadas, además de recomendaciones generales para otras playas en los dos países. Se destaca la necesidad de un proceso de gestión de playas caracterizado por ser local, público, participativo, formalizado e integrado.

Palabras-clave: gobernanza; gestión integrada de las zonas costeras; gestión de playas.

RESUMO: Tradicionalmente, a gestão de praias não tem fomentado a governança, aspecto essencial para a sustentabilidade de tais ambientes costeiros, dado que promove ações para o bem comum. Este trabalho tem como fim entender e comparar os processos de governança na gestão das praias Pocitos (Montevidéu, Uruguai) e Central (Balneário Camboriú - SC, Brasil). Os objetivos propostos foram caracterizar o arcabouço institucional para a gestão de praias em ambos os casos, os stakeholders envolvidos e os resultados alcançados, sugerindo ajustes às ferramentas e aos desenhos atuais. Entrevistas semiestruturadas foram realizadas e classificadas em seis componentes da governança. A instituição responsável pela gestão das praias e os demais stakeholders foram aproximados por intermédio da técnica da Bola de Neve. Na praia de Pocitos, as atividades de gestão são realizadas por meio de um processo participativo e integrado, principalmente envolvendo divisões do governo, que conformam um comitê de trabalho semanal, que permite a implementação dos processos de governança. Porém, reformas são necessárias, especialmente relacionadas à participação de um amplo leque de stakeholders. Na praia Central, as atividades de gestão demonstram uma integração débil entre as divisões do governo municipal e a participação de outros stakeholders não é usual. Uma alternativa de gestão similar à de Pocitos é sugerida para a praia Central. A contribuição principal deste trabalho está na proposta de um conjunto de recomendações para a gestão de ambas as praias analisadas, além de recomendações gerais para outras praias nos dois países. Destaca-se a necessidade de um processo de gestão de praias caracterizado por ser local, público, participativo, formalizado e integrado.

Palavras-chave: governança; gestão integrada das zonas costeiras; gestão de praias.

ABSTRACT: Traditionally, beach management has not encouraged governance, which is essential for the sustainability of these coastal environments, as it promotes actions for the common good. This work aims to understand and compare the governance processes in the management of Pocitos beach (Montevideo, Uruguay) and Central beach (Balneário Camboriú-SC, Brazil). The goals were to characterize the institutional framework of the beach management, the stakeholders involved, and the results achieved in both cases suggesting adjustments to the current designs and tools. Semi structured interviews based on six components of governance were conducted and classified. The institution responsible for beach management and the stakeholders were approached via Snowball Sampling technique. On Pocitos beach, management activities are performed through a participative and integrative process, mainly involving government divisions that conform a committee meeting weekly. This committee allows the implementation of governance processes. However, improvements are needed, especially in terms of participation of a wider range of stakeholders. On Central beach, management activities show a weak integration between the municipal government divisions, and the participation of stakeholders. A management alternative similar to that of Pocitos beach is suggested for Central beach. The main contribution of this work is the proposal of a recommendations set for the management of the two beaches addressed, as well as a generalization for other beaches in both countries. We highlight the need for beach management processes characterized by being local, public, participatory, formalized and integrated.

Keywords: governance; integrated coastal zone management; beach management. 


\section{Introducción}

En las últimas décadas, la presión humana ha sometido a las zonas costeras del planeta y sus recursos a dinámicas de destrucción del patrimonio natural, erosión generalizada, urbanización y masificación creciente (IPCC, 2014). Las playas en particular están amenazadas por presiones antrópicas intensas, tales como actividades recreativas y cambio climático, resultando en múltiples impactos (Defeo et al., 2009). En muchos municipios costeros, en lugar de manejar las actividades impactantes, se percibe un nivel de gestión de playas aún débil, centrado básicamente en limpieza y primeros socorros, con planeamientos urbanísticos para la zona litoral y sus accesos que deberían ser mejorados. Además, generalmente son escasos los recursos invertidos para la gestión de playas, la que a veces no es asumida por ningún departamento específico de la administración (Yepes, 2007).

Las playas son el lugar donde los turistas evalúan la calidad de la oferta turística de la ciudad costera, los espacios más representativos y fotografiados a nivel turístico, y deben ser concebidas como generador económico, por lo cual también deben ser estimadas por otros atributos valiosos, como la protección costera y el valor ambiental (Yepes, 2005). En la sociedad postindustrial, la población residente, que tenía en su ciudad convencional un centro de residencia y trabajo, empieza a ver a la playa como generadora de espacios de ocio (Anton, 1998). La dinámica turística y residencial ha influido en la masificación del uso de sol y playa, intensificado a partir de la década de 1960 del siglo XX (MinTur, 2010).

Como respuesta, la gestión de playas - entendida como un proceso de gestión costera pragmático, especifico y local (Williams \& Micallef, 2009)
- se ha convertido en una cuestión cada vez más evidente en la agenda política de los países industrializados, desde que se creó el primer esquema de certificación de playas (Bandera Azul) en Europa, en los años 1980s (Botero, 2009). Actualmente, existe un amplio rango de certificaciones para la gestión de playas (ej. Bandera Azul y ISO 14.001), cada una representando abordajes muy diferentes (Williams \& Micallef, 2009). Recientemente, se ha reconocido la necesidad de evolucionar hacia la gobernanza costera (Olsen \& Ochoa, 2007), ya que ésta y por consecuencia la gobernanza de playas, tienen como objetivo gobernar las actividades humanas, los comportamientos y los recursos ocurrentes en las zonas costeras, por medio de estructuras y procesos, realizados por el Estado, el sector privado y la sociedad civil (Ehler, 2003).

En Latinoamérica, dónde todavía hay una carencia de inversiones, herramientas y oportunidades para el desarrollo sostenible de las costas (Ittekkot, 2015), la gestión de las playas solo se convirtió en una realidad en 2003, cuando Uruguay implementó la certificación Playa Natural (Botero, 2009), seguido por el establecimiento de un Sistema de Gestión Ambiental (SGA) para las playas de Montevideo, basado en la Norma ISO 14.001. Este sistema abarca cinco temas principales: servicios, infraestructura, conservación, recreación y otros (Marchese et al. 2013), y se basa en tres compromisos: mejora continua del sistema, prevención de la contaminación y cumplimiento de la legislación y otros reglamentas (AENOR, 2014). El SGA se ha convertido en un modelo potencial para sitios similares.

El presente artículo analiza los procesos de gobernanza en la gestión de las playas Pocitos (Montevideo, Uruguay) y Central (Balneário Camboriú - SC, Brasil) en base a una caracterización del andamiaje institucional para la gestión en ambos 
casos, los stakeholders presentes, y los resultados alcanzados, desarrollando recomendaciones generales para el ajuste del diseño y de la aplicación de herramientas de gestión para ambas localidades. El objetivo final es contribuir al establecimiento de recomendaciones para la gestión de playas de Uruguay y Brasil, en una escala nacional.

\section{2. Área de estudio}

El área de estudio incluyó a las playas Pocitos (Montevideo, Uruguay) y Central (Balneário Camboriú - SC, Brasil) (Figura 1), que pueden
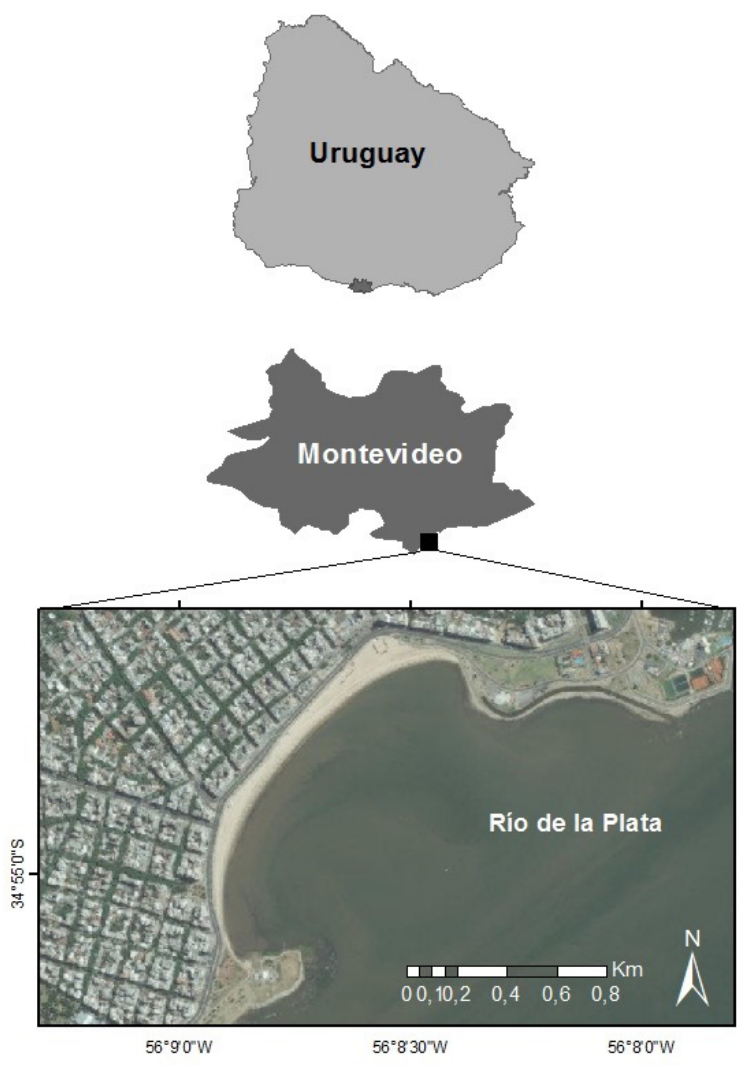

FIGURA 1 - Ubicación de las playas Pocitos (Montevideo, Uruguay) y Central (Balneário Camboriú - SC, Brasil). 
por algunas áreas específicamente designadas en Brasil (Brasil, 2004).

En cuanto a la división política, tanto Uruguay como Brasil poseen tres niveles de gobierno, pero algunas diferencias son relevantes. En Uruguay, las estructuras de gobierno se han concentrado históricamente en el Departamento de Montevideo, dónde vive aproximadamente la mitad de la población del país (INE, 2011). En Montevideo, la descentralización al nivel municipal empezó solamente en los años 1990s, con la creación de centros zonales (Veneziano, 2008), los cuales hasta el momento tienen más características de organismos desconcentrados del Intendente, que de organismos descentralizados (Gutiérrez, 2013). En Brasil, el gobierno municipal ya presenta una autonomía considerable en lo que refiere al planeamiento urbano, conferida por el federalismo (Frey, 2012), y el municipio de Balneário Camboriú posee una historia más larga que tiene raíz en su establecimiento en el año 1964 (PMBC, 2013).

Con respecto a la estructura normativa para la zona costera, Uruguay todavía no presenta una legislación específica, aunque sí existen otras leyes relacionadas al tema. En Montevideo, siete playas están certificadas por la Norma ISO 14.001, más allá de algunas puntas rocosas y áreas verdes. A su vez, Brasil presenta un conjunto de leyes específicas para la gestión costera (Brasil, 1988a; Brasil, 2004), entre otras leyes relevantes al tema (Andrade \& Scherer, 2014). Con respecto a la gestión de sus playas, un instrumento público para el ordenamiento de la orilla - Projeto Orla - ha sido estructurado (Brasil, 2004), por el cual se han formulado planes para cerca de un quinto de todas los municipios costeros brasileños, no obstante no hay aún información sobre su real implementación (Oliveira \& Nicolodi, 2012). Además, cuatro playas brasileñas poseen la certificación Bandera Azul.
Ambas herramientas han encontrado dificultades en su implementación en la costa brasileña (Scherer, 2013), y ninguna de ellas ha sido empleada en Balneário Camboriú.

Pocitos es la playa más concurrida de Montevideo, estando ubicada en un importante barrio residencial, cercano al centro de la ciudad. Es muy popular para la práctica de deportes terrestres y acuáticos, y su paseo marítimo es usado para la circulación y para la contemplación del paisaje (IDM, 2013b; MTD, 2014). Como consecuencia de su SGA, exhibe buena infraestructura, la cual permite el disfrute de la playa por turistas y residentes (IDM, 2013a). Sin embargo, ha presentado algunos problemas de erosión, por lo cual se han realizado rellenos de arena desde de la década de 1970 (Gutiérrez, 2010).

De forma similar, la playa Central es la playa más concurrida de Balneário Camboriú, recibiendo un alto flujo de turistas en la temporada de verano - cerca de 1.5 millones de personas (Costa et al., 2006) - en contraste con su población residente de 110.748 habitantes (IBGE, 2011). Su rápido crecimiento urbano, experimentado desde los años 1970, sin un proceso de planeamiento integrado efectivo, ha producido innumerables problemas y conflictos urbanos y ambientales, como la degradación de ecosistemas naturales y el transito caótico en los meses de verano (Skalee \& Reis, 2008). Asimismo, no cuenta con un proceso de gestión formalmente establecido, si bien se verifican algunas actividades realizadas en el ámbito de las playas (Bombana \& Polette, 2012).

\section{Metodología}

Entrevistas semi estructuradas (Batthyány et al., 2011) fueron conducidas con stakeholders de 
ambas playas, determinados como todos los actores involucrados, interesados en y/o afectados, de forma positiva o negativa (Pomeroy \& Douvere, 2008) por diversas acciones llevadas a cabo en las playas. Como actores involucrados, se han definido aquellos que presentan responsabilidades en decidir y realizar los procedimientos/actividades de gestión (limpieza de la arena, muestreos de calidad del agua, salvamento acuático, etc.).

A lo largo de las entrevistas, un conjunto de cuestiones ha sido abarcado (Figura 2), de forma libre, basado en otros abordajes y estudios sobre gobernanza (Whittingham, 2005; Olsen \& Ochoa, 2007; Cheong, 2008; Albuquerque et al., 2008). La mayoría de los entrevistados expresó sus opiniones desde la perspectiva de la institución que estaban representando, aunque algunas opiniones personales también fueron detectadas.
Para conformar la cadena de entrevistados, se empleó la técnica de la Bola de Nieve, que recomienda un primer contacto con una persona relacionada con la problemática, la cual es invitada a indicar otros contactos también relacionados. El universo de muestreos es establecido cuando la mayoría de las personas indicadas empiezan a ser repetidas, finalizándose el proceso con un listado de entrevistados potenciales (Wright \& Stein, 2005). Los trabajos de Marchese et al. (2013) y Bombana \& Polette (2013), sirvieron como complemento para aclarar el arreglo de instituciones involucradas con la gestión en cuestión. Algunos de los stakeholders, aunque listados, no pudieron ser consultados por razones particulares.

El marco analítico combinó las entrevistas con elementos descriptivos y normativos, con los resultados de estudios anteriores sobre la temática

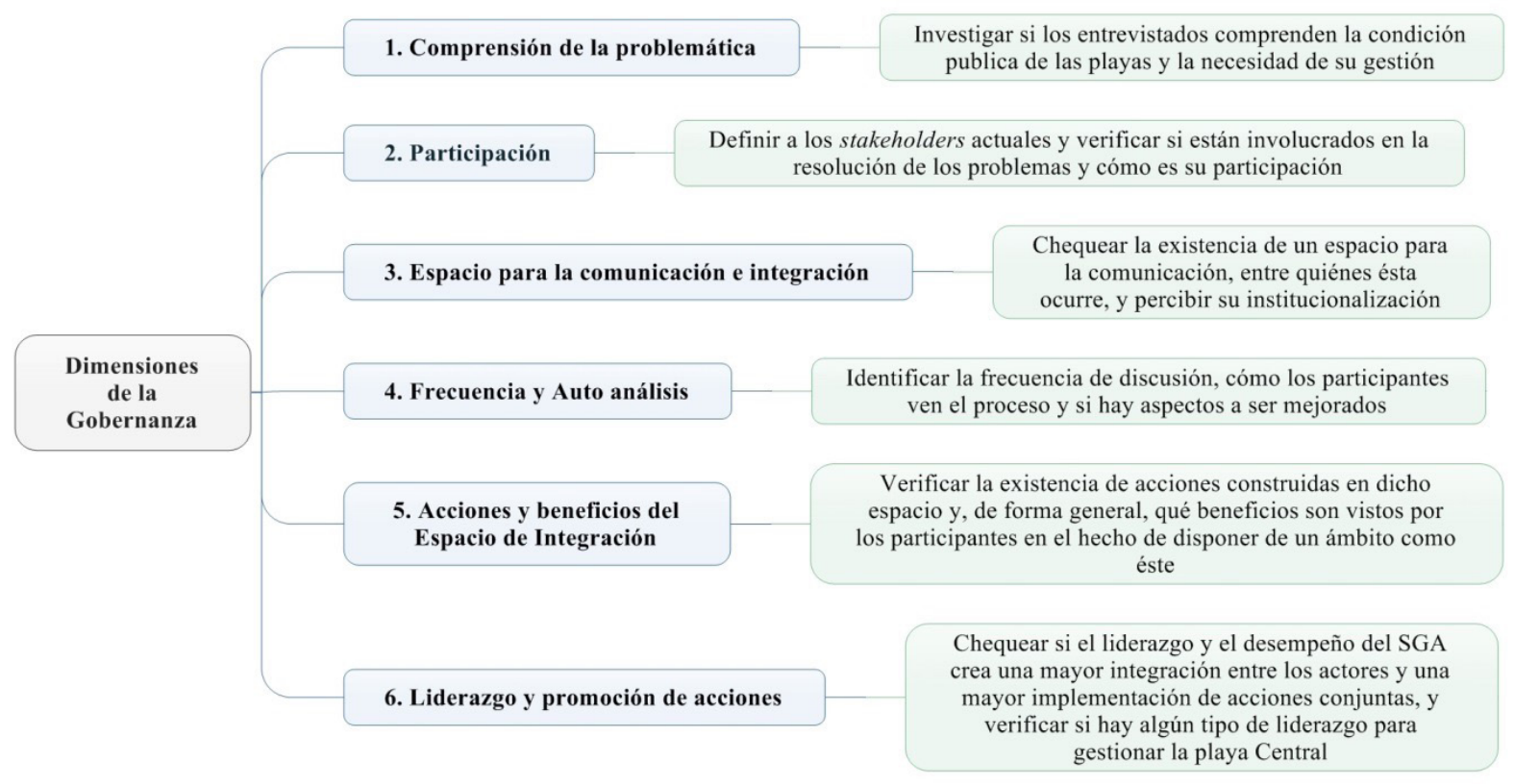

FIGURA 2 - Dimensiones de la gobernanza y sus objetivos respectivos, considerados en el marco de las entrevistas. 
y sitios similares, y el conjunto actual de leyes y otros acuerdos. La transcripción y la clasificación de los entrevistados fueron realizadas por medio de un análisis cualitativo de contenido, basado en componentes de gobernanza previamente definidos. Para apoyar el análisis comparativo, se consideró al índice de calidad de gobernanza (ICG), que es utilizado para definir el grado de coordinación local de los agentes implicados en proyectos concretos con impacto en las playas (Valls et al., 2014). Se optó por emplearlo cualitativamente, apenas con el fin de destacar los temas que determinan a dicho grado de coordinación.

\section{Gobernanza en la Gestión de las Playas de Pocitos y Central}

\subsection{Andamiaje institucional}

Para la playa de Pocitos, un total de 21 stakeholders $^{1}$ directamente relacionados con la playa fueron listados (Tabla 1), de los cuales 16 fueron entrevistados como representantes de instituciones (o de subdivisiones de instituciones). Del total, solamente 7 se involucran directamente y activamente en los procesos del SGA, siendo 6 divisiones del gobierno departamental, y 1 institución del gobierno nacional.

La distribución de las responsabilidades de gestión casi unánime en la Intendencia Departamental de Montevideo (IDM) es atribuida al hecho de que ésta ha sido tradicionalmente la institución responsable por la administración pública en el departamento de Montevideo y sus municipios, y también porque la legislación montevideana asigna competencias específicas a la IDM con respecto al cuidado y protección de las playas, así como de los paseos y calzadas de ríos (Biasco, 1999). Sumado a esto, fue a partir de una iniciativa surgida dentro de la Unidad de Gestión de Calidad de la IDM que se ha instituido el SGA, apoyando la idea de que los actuales sellos de calidad de playas latinoamericanas presentan un fuerte abordaje de arriba hacia abajo (Botero, 2012).

En Pocitos, se destaca la ausencia de la sociedad civil y del sector privado, aunque la primera puede ser incorporada por el mecanismo de representación ciudadana - Consejo Vecinal (Uruguay, 2009) - que es una forma de asegurar los aportes de la sociedad civil en el proceso. De hecho, para otras playas montevideanas (Malvín y Buceo), ubicadas en un municipio diferente del que corresponde a Pocitos, el Consejo Vecinal tiene representantes de la sociedad en la dinámica del SGA.

Para la playa Central se estableció un listado de 35 stakeholders directamente relacionados con la playa (Tabla 1), de los cuales 22 fueron entrevistados, representantes de instituciones (o de subdivisiones de instituciones). Del total, 15 actúan directamente en los procesos informales de gestión, los cuales son integrantes del poder público, en su mayoría municipal. Igualmente, se ha observado una cierta diversidad en el listado, que integra el sector privado, la sociedad civil, más allá de otros niveles de gobierno (nacional y estadual).

De forma similar al caso montevideano, se percibió un predominio de responsabilidades de gestión de las playas distribuidas en el gobierno, particularmente el municipal, aunque la competencia legal para ello está atribuida a la Secretaria

\footnotetext{
1 Un stakeholder representa a un grupo, institución u organización individual, excepto para las instituciones del gobierno (en los tres niveles), en las cuales un entrevistado representa a una de sus divisiones, independientemente de la existencia de otras subdivisiones en la misma.
} 
TABLA 1 - Listado y clasificación de los stakeholders relacionados con la problemática de gestión de las playas en Pocitos (Uruguay) y Central (Brasil).

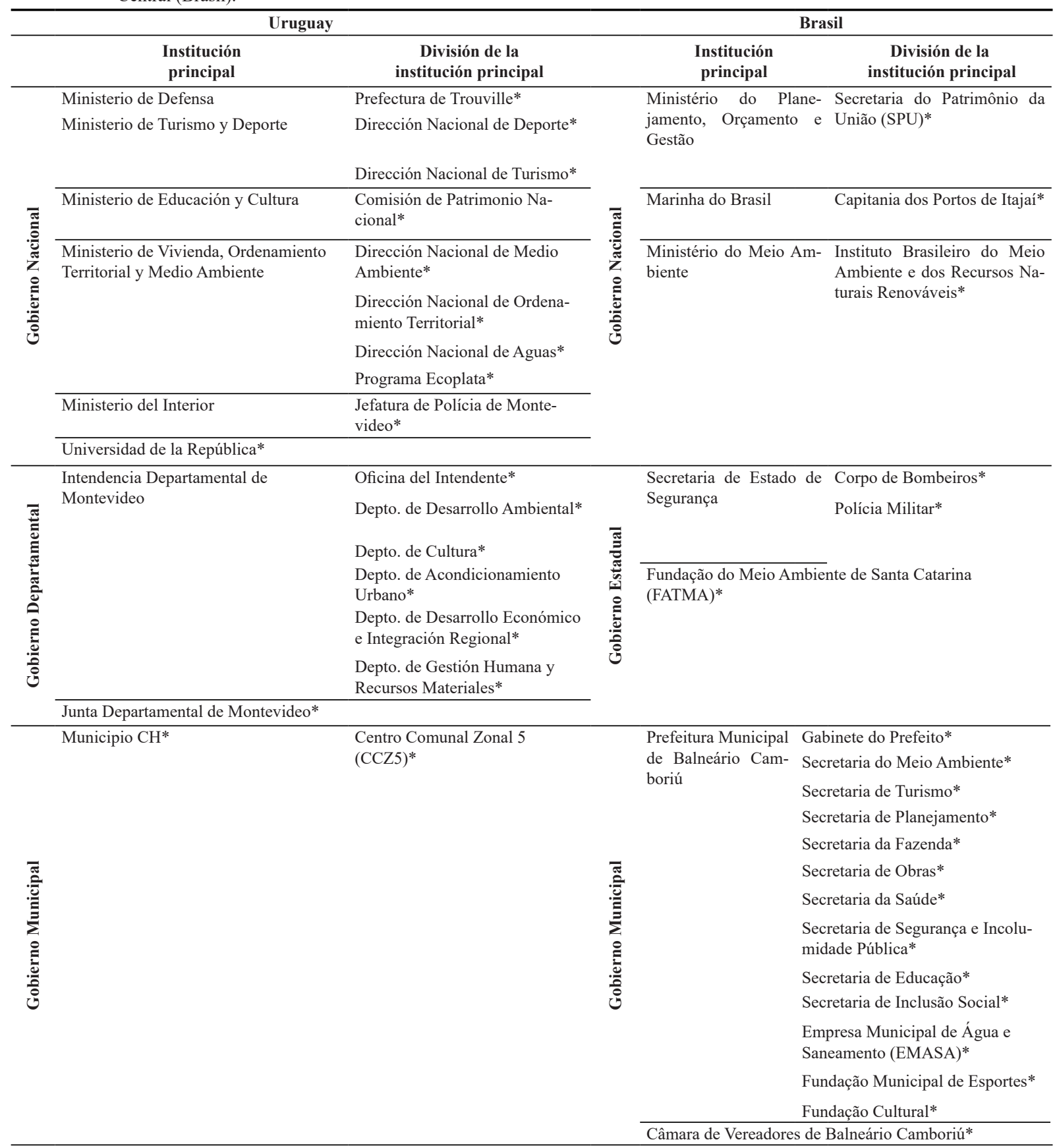

(continua) 
Associação de Bodyboarding de Balneário Camboriú (Acambody)*

Liga Independente das Canchas de Bocha*

Associações das Canchas de Bocha da Praia*

Câmara de Dirigentes Lojistas (CDL)*

Sindicato de Hotéis, Bares, Restaurantes e Similares de Balneário Camboriú e Região (Sindisol)*

Sindicato dos Empregados do Comércio Hoteleiro, Bares,

Restaurantes e Similares de Balneário Camboriú

(Sechobar)*

Sindicato da Indústria da Construção Civil (Sinduscon)*

Federação Catarinense de Surf (Fecasurf)*

Associação Empresarial de Balneário Camboriú e Camboriú (Acibalbc)*

Associação dos Corretores de Imóveis de Balneário Camboriú (ACIBC)*

Convention Visitors \& Bureau*

Empresa Geo Consultores*,

\footnotetext{
* Stakeholders directamente mencionados a lo largo del empleo del conjunto de metodologías para conformar la cadena de entrevistados; a - Incluye también a la sociedad civil no organizada: Turistas, residentes, vendedores ambulantes, vendedores de bares en las playas, etc.; b - Incluye también a los proveedores privados, no considerados en el proceso porque son contratados y coordinados por el gobierno; c - Opera cuando los procesos de certificación y acreditación son llevados a cabo.
}

do Patrimônio da União (SPU). Se infirió que lo observado ocurre debido a la autonomía de los municipios brasileños en la gestión urbana (Frey, 2012), a la carencia de recursos por parte de la SPU y por el hecho de que es en esta escala que los problemas son percibidos, siendo necesario, cuando es posible, intervenciones inmediatas para resolverlos. Los demás tipos de instituciones (sociedad civil, privados y otros niveles de gobierno) no se involucran en la gestión de la playa, aunque tienen interés, afectan o son afectadas por los problemas o temas relevantes.
La predominancia tanto de la IDM en el SGA en Pocitos, como del gobierno municipal en la gestión informal de Central, es una limitante a la práctica de gobernanza, la cual debe contemplar la mayor diversidad de actores posibles relacionados a la problemática (acercados y representados en la Tabla 1). Adicionalmente, los actores sin representación organizada (ej. turistas y vendedores ambulantes) son un componente clave que, siendo usuarios de los espacios playa, poseen preferencias y hábitos variables (Vaz et al., 2009). Todas esas partes deberían ser, por lo tanto, idealmente incluidas en los procesos de gestión. 


\subsection{Análisis de los componentes de gobernanza}

\subsubsection{La comprensión del problema}

La comprensión del problema abarcó las siguientes cuestiones: Derecho de Propiedad, siendo las playas de Pocitos y Central clasificadas como de dominio público, pertenecientes a la Nación (Biasco, 1999; Brasil, 2004); Competencias de Gestión, las cuales destacan la responsabilidad del gobierno en todos sus niveles, si bien la sociedad también debe jugar un papel importante (Uruguay, 2009; Brasil, 1988b); y, de la comprensión de la necesidad de su gestión, ya que las playas desempeñan múltiples funciones que deben ser mantenidas, como recreación, defensa costera y conservación (Williams \& Micallef, 2009).

La comprensión del derecho de propiedad de las playas y a quiénes compete la gestión de las mismas se mostró intermedia para el caso uruguayo y baja para el caso brasileño, asociado a la existencia o inexistencia de una gestión formalizada. Se infirió que en el caso uruguayo no ha sido más alta porque no todos los entrevistados son parte integrante del SGA, el cual contribuye a que las partes responsables tengan más claras las cuestiones referentes a las playas, y porque algunos aún interpretan a las intervenciones de gestión como de responsabilidad total de la IDM.

Según Berkes \& Folke (1998), los derechos de propiedad son un elemento clave para mejorar el manejo de los recursos (en este caso, el sistema playa), visto que el poseedor del derecho empieza a presentar una mayor noción de pertenencia y de responsabilidad, que a su vez, garantiza la participación y proporciona legitimidad al proceso de manejo. En el contexto de territorios pertenecientes a la Nación, se destaca la importancia de comprender los regímenes de propiedad, ya que éstos también pueden ser inter- pretados como un elemento clave para el manejo. En ambos casos considerados, todos los entrevistados vieron a las playas como espacios de uso público.

Por otro lado, la comprensión de la necesidad de gestión de las playas por los responsables actuales de llevar a cabo los procedimientos de gestión fue alta para el caso uruguayo y baja para el caso brasileño, nuevamente condicionada a la existencia de un proceso de gestión formalizado. Se destaca que en la playa brasileña, las actividades son llevadas a cabo de forma puntual y sectorial por las secretarias municipales, principalmente de acuerdo a las necesidades impuestas por la temporada estival. En contraste, en el caso montevideano, la temporada baja es considerada un periodo importante para la gestión, principalmente debido a los temporales y a las acciones de recuperación y mantenimiento, realizadas con vistas a "preparar" las playas para el verano.

\subsubsection{La participación}

Pomeroy \& Douvere (2008) defienden que existen distintos niveles (del más bajo al más alto) de participación (comunicación, información, consulta, diálogo, concertación y negociación) algunos de los cuales pueden reforzar procesos de dominación, mientras que otros pueden promover la emancipación de los sujetos participantes.

Para la gestión de la playa de Pocitos, la participación, más allá de la mera comunicación e información, ocurre principalmente por la participación de las divisiones de la IDM, que llevan a cabo procedimientos en las playas y se encuentran en un comité participativo (CP). Se observó que la participación de los demás stakeholders (sociedad civil, privados, y otros niveles de gobierno) con respecto al listado conformado en ese trabajo (Tabla 1) ocurre de forma débil o ausente, lo que deja 
la gestión vulnerable ante intereses particulares (Marchese, et al., 2013). Sin embargo, se percibe que entre el CP y los demás actores los dos primeros niveles de participación pueden ocurrir, siendo éstos los únicos requeridos por la Norma ISO 14.001.

En la gestión informal de la playa Central prevalecen como participantes las instituciones gubernamentales de nivel local. Debido a que cada institución actúa de manera independiente (cooperativamente, solo en pocos casos puntuales) en el desarrollo de procedimientos de gestión, no está claro si existe o no una lógica de participación conjunta. Esta dinámica resulta similar a lo observado en algunas playas españolas, donde su gestión conserva la fragmentación de sus funciones en muchas oficinas y la administración realizada por actores con responsabilidades muy distintas, lo que se constituye en un impedimento para el uso sostenible (Valls et al., 2014).

Williams \& Micallef (2009) convergen sobre la gobernanza costera al afirmar que la promoción de la participación es axiomática para la gestión de las playas como zonas complejas, visto que una persona no puede comprender todas las demandas hechas para estos espacios, siendo el esfuerzo conjunto sustancialmente necesario. En el momento de considerar y promover un esfuerzo conjunto, es vital percibir la participación a partir del diálogo entre los múltiples stakeholders, aunque la información y la comunicación también sean mecanismos importantes para otros aspectos, como informar sobre los servicios ofrecidos.

\subsubsection{El Espacio de Comunicación e Integración}

En el caso de Montevideo, se infiere que la centralización de los procedimientos del SGA generó la necesidad y formalizó la existencia de un espacio para la comunicación, discusión e integración de acciones, que es el CP. En Balneário Camboriú, a su vez, no existe un espacio de esta naturaleza para la gestión de sus playas.

El CP, específicamente, está compuesto no solo por las instituciones que concurren a sus reuniones periódicamente, sino que también tiene un espacio físico determinado dentro de la IDM que no es atribuido a ninguna oficina específica, convirtiéndose en un organismo autónomo. El CP promociona la integración entre el grupo mencionado, y presenta el potencial de incrementar todos los tipos de participación, para el conjunto total de stakeholders, al existir una motivación interna de invitar a las reuniones y divulgar sus actas a todos. En la política del SGA fueron establecidas acciones para garantizar la coordinación entre los sectores y promocionar el involucramiento y el apoyo de la población residente (IDM, 2012).

Para promocionar el involucramiento de todos los stakeholders en las decisiones es necesario permitir su empoderamiento, hecho también mencionado en la política del SGA. El objetivo final de estas actividades es establecer cambios en la actitud general, para tener un proceso de gestión sustentable en el tiempo. Tanto la participación como el empoderamiento demandan tiempo y recursos (Pomeroy \& Douvere 2008) y deben ser trabajados independientemente de las voluntades contrarias de algunos miembros del ámbito.

Para Balneário Camboriú, la inexistencia de un sistema y un órgano para la gestión de la playa Central, así como de un espacio para la comunicación e integración, resaltan su necesidad. Zielinski \& Botero (2012) llaman a la necesidad de constituir un órgano gestor de playas como uno de los requisitos primarios para la gestión ambiental y posterior certificación. Algunos de los entrevistados han 
sugeridos que este órgano debería ser integrado a un comité ya existente para la resolución de cuestiones municipales críticas, como es el comité de la cuenca hidrográfica, pero su factibilidad necesita ser confirmada, y puede no ser necesaria si un SGA llega a ser implementado en este municipio.

\subsubsection{Frecuencia y Autoanálisis del Espacio de Integración}

A pesar de la frecuencia variable de participación de los diferentes stakeholders en el CP, las reuniones son mantenidas semanalmente. De hecho, la presencia semanal en el CP de todos puede no ser necesaria o factible (ej. la Prefectura de Trouville solo concurre algunas pocas veces al ámbito, justificado por una carencia de recursos humanos y por ser responsable de actividades desarrolladas en una amplia parte del territorio). Algunos stakeholders pueden tener su presencia requerida solamente en casos y/o en tiempos específicos (ej. Dirección Nacional de Deporte influye en la playa Pocitos solamente durante el verano, cuando instala un estadio en la faja de arena). En este sentido, se debe emplear mecanismos o metodologías para garantizar la participación, aunque con frecuencias distintas, de acuerdo a las realidades de cada institución y/o caso.

En lo que se refiere al auto análisis, se apreció una satisfacción general de los participantes consultados con la gestión de la agenda, la frecuencia, la asiduidad, la estabilidad del ámbito y de los participantes. Esto fue inferido como producto de la estructura institucional, normativa (con respecto a la ISO 14.001) y física (ej. Salón de reunión) que se construyó y se mantiene en la IDM desde la institución del SGA. Sin embargo, estas respuestas corresponden a los participantes del CP y pueden evidenciar una característica tendenciosa.
Los aspectos a mejorar, según las opiniones recabadas, incluyen el compromiso de los stakeholders y la comprensión de su rol en el sistema; la variabilidad de la asiduidad de los participantes y la ausente o débil participación de actores de otra naturaleza; la limitación de la participación pública, por ser apenas una comunicación de las decisiones; la debilidad de los términos de comunicación interna y externa; a la necesidad de asegurar los recursos por medio de un presupuesto propio y de crear una mayor capacidad para adaptarse más a los cambios positivos en la estructura política (ej. los municipios instituidos aún no están integrados a la dinámica del comité). Éstos aspectos deben ser incorporados a la premisa de mejoría continúa de la ISO 14.001, considerando el carácter dinámico de estos ambientes y de los propios stakeholders en sus interacciones con el medio, que tienden a cambiar constantemente en el tiempo y espacio, debido a influencias externas e internas.

Para mejorar e incrementar la concurrencia de los stakeholders al ámbito se ha sugerido que éste posea una carga horaria fuera del horario laboral (especialmente cuando se trate de asuntos muy complejos), para que la mayor parte posible, más allá de la IDM, pueda concurrir a la dinámica. También se ha sugerido facilitar una estructura física propicia a la participación (como ejemplo, disponer de un salón adecuado al conjunto posible de representantes), y más en general a mejorar la comprensión de los objetivos y las necesidades de la gestión de las playas.

Las sugerencias para incrementar la concurrencia al ámbito mediante la motivación de los participantes actuales del CP demuestra una relación con procesos de empoderamiento, ya que para el SGA ha desarrollado actividades (ej. talleres) con la intención de aclarar la importancia del sistema y de la costa, y capacitar a los actores para realizar sus 
actividades. Poitras et al. (2003) identifican la falta de entendimiento de la importancia del proceso y de las reglas para la gestión de los recursos como uno de los diez desafíos a la construcción de consenso en los procesos de gestión costera integrada.

\subsubsection{Acciones y Beneficios del Espacio de Integración}

Los beneficios del CP se refieren fundamentalmente a: que la implementación del SGA ha promocionado la toma de conciencia y discusión conjunta entre las partes de la IDM y sus proveedores acerca de la importancia de la gestión de las playas; la minimización más factible de los conflictos; una mirada menos sectorial sobre los procesos costeros, debido a un aporte de distintas visiones que enriquece la forma de resolver las cuestiones pertinentes; un mejor reglamento de actividades previas a su implementación; una promoción de nuevas actividades, también reguladas por el SGA; una sistematización de todas estas tareas en las playas. Recíprocamente, puede resaltarse que el SGA se ha expandido hacia otras zonas de la costa montevideana desde su implementación, el CP se hizo más respetado por las reparticiones de la IDM $\mathrm{y}$, en encuestas realizadas con la población, se ha verificado que la costa está mejor después de la implementación del sistema.

Dentro de las acciones desarrolladas, se destaca: la maximización de los recursos disponibles; la planificación y operación conjunta; una más rápida e integrada resolución y comunicación de los pro- blemas; la información en tiempo real del estado de las playas y el rastreo más fácil de las acciones; el reglamento y cumplimiento de normativas para las actividades. Éstas tienen correspondencia con los requisitos establecidos en la ISO $14001^{2}$, los cuales abarcan los cuatro componentes clave relevantes para una gestión estratégica de las playas (Micallef \& Williams, 2002): Análisis, Planeamiento, Manejo y Monitoreo. Además, en los países como Uruguay, dónde aún hay una carencia de normativas legales para la gestión costera integrada, las certificaciones ambientales voluntarias sobre temas costeros facilitan la creación y la formalización de espacios para la resolución de los problemas.

El análisis de la aplicabilidad de la certificación ISO 14.001 es relevante cuando se considera la ausencia de acciones integradas en la gestión de playa Central y, especialmente, la débil y difícil implementación de iniciativas específicas para las playas brasileñas (Scherer, 2013). Zielisnki \& Botero (2012) argumentan que certificaciones voluntarias traen beneficios tales como la adopción de prácticas apropiadas de conservación, y el reconocimiento y la diferenciación de la playa como un producto turístico.

La situación de Balneário Camboriú recibe una importancia destacable, ya que un plan dentro de los moldes del Projeto Orla fue formulado ya en 2004, pero nunca ha sido implementado debido a una falta de voluntad política. A su vez, al presentarse la certificación ISO 14.001 a algunas autoridades $^{3}$ del poder municipal ejecutivo y legislativo, se percibió que éstas se quedaron interesadas en

\footnotetext{
2 Requisitos de la Norma ISO 14.001: 1. Requisitos Generales; 2. Política Ambiental; 3. Planificación; 4. Implementación y Operación; 5. Verificación y Acción Correctiva; y, 6. Revisión por la Dirección (AENOR 2014).

3 Los autores presentaron la certificación ISO 14.001 como una alternativa para la gestión de las playas de Balneario Camboriú-SC, Brasil. La presentación tuvo lugar en el órgano legislativo del municipio - Câmara Municipal de Vereadores - en la fecha 01/07/2014. Más información disponible en: <http://www.cambc.sc.gov.br/documentos/textoproposicao/20140574>.
} 
llevarla a cabo en el futuro, principalmente porque la certificación puede ayudar a generar una publicidad positiva para el municipio.

\subsubsection{Liderazgo y Fomento de Acciones en el Espacio de Integración}

El liderazgo en el SGA ocurre a través del coordinador del CP (coordina los stakeholders actuantes y las acciones en las playas) y de la autoridad máxima del sistema (toma la decisión final en lo que se refiere a la gestión de las playas), delegado por la autoridad máxima de la IDM. De esa forma, entender un buen liderazgo para este sistema supone que la comunicación entre ambos sea fluida y positiva, en lo que refiere a las acciones planeadas y emprendidas.

Los participantes del CP entrevistados defendieron que la capacidad de su coordinador para liderar la gestión de las playas certificadas es satisfactoria. Se ha verificado que el CP y su coordinador favorecen la condición organizacional propicia a una mejor interacción entre los actores y una mayor realización de acciones comunes, hechos asumidos como capacidades de un liderazgo eficaz (Bergamini, 1994). Se ha percibido también la existencia de características importantes a ser desarrolladas por un gestor costero (Shah et al., 2007), al cual debe corresponder una buena capacidad de liderazgo. Estas características son la habilidad de comunicar e interpretar información de y para una variedad de audiencias, y la destreza en la gestión de costos, tiempo y proyectos en marcha.

Efectivamente, se puede interpretar como indicador de la capacidad de liderazgo el hecho de que aunque lo definido en el CP no sea obligatorio, acaba siempre cumplido por las entidades de la IDM y es mayormente cumplido por las entidades no componentes del gobierno departamental, cuando a éstas se les informa cómo deben proceder con sus actividades.

Finalmente, en la Tabla 2 se presenta el análisis de las dimensiones de gobernanza en formato de indicadores del proceso actual encontrado para cada una de las playas. El análisis comparativo ha demostrado que los procesos de gestión y de gobernanza en las playas de Montevideo (especialmente Pocitos) se encuentran en un nivel más avanzado que en la playa Central, si bien todavía hay margen de mejora para ambas ciudades.

\subsection{Recomendaciones de Ajustes al Diseño y a la Aplicación de Herramientas}

Valls et al. (2014) definen la gobernanza como el factor más decisivo para la calidad recreativa de las playas y propusieron el índice de calidad de gobernanza (ICG) como forma de medirla. Los ítems del ICG han sido considerados cualitativamente, con el fin de apoyar el estudio comparativo entre las playas estudiadas (Tabla 3). Los resultados convergen hacia la existencia de un proceso más avanzado en Montevideo en las cuestiones de gobernanza en comparación con Balneario Camboriú.

En la Tabla 3, los ítems que forman el ICG están listados en la primera columna. De una forma general, a pesar del mayor avance de Montevideo en la gestión de playas, se percibió que ambas ciudades presentan planes urbanos que incluyen cuestiones urbanas y turísticas relacionadas a las playas y su entorno. Sin embargo, los procedimientos actuales de gestión de playas son condicionados a la existencia de un plan específico para las mismas, que en el caso de Montevideo - la política ambiental del SGA - ha llevado a una certificación ambiental. Hay también una carencia de información respecto a ciertas mate- 
TABLA 2 - Resumen de indicadores del proceso de gobernanza en las playas Pocitos y Central.

\section{Indicadores de gobernanza local}

Número de stakeholders relacionadas con la problemática de las playas

Número de participantes frecuentes y directos en las intervenciones de gestión

Correspondencia entre las instituciones responsables por realizar procedimientos de gestión (formal o informal) en la playa, con las partes interesadas, afectadas o que la afectan de alguna manera

Entendimiento del derecho de propiedad de las playas y a quiénes compete la gestión de las mismas

Comprensión de la condición pública de las playas

Comprensión de la necesidad de gestión de las playas por los responsables de los procedimientos de gestión

Participación en los procedimientos de gestión (formal o informal)
Playa de Pocitos

21

7

Baja Baja

\begin{tabular}{cc}
\hline Mediana & Baja \\
\hline Alta & Alta \\
\hline
\end{tabular}

Alta

Baja y enfocada en la temporada estival

Participación gubernamental:

Básicamente departamental

Participación pública: Ausente

Participación de privados: Ausente
Playa Central

34

15

Participación gubernamental: Básicamente municipal

Participación pública: Ausente

Participación de privados: Ausente

\begin{tabular}{|c|c|c|}
\hline \multirow{3}{*}{ Espacio de comunicación para los temas de la playa } & Existente & Ausente \\
\hline & Formalizado a través del SGA & Sin formalidad \\
\hline & $\begin{array}{c}\text { Participantes, esencialmente la IDM, } \\
\text { esencialmente la IDM }\end{array}$ & Sin participantes \\
\hline \multirow{3}{*}{ Encuentro en el espacio de comunicación } & Frecuencia Semanal & Inexiste \\
\hline & Auto análisis adecuado & Inexiste \\
\hline & Aspectos a mejorar & Inexiste \\
\hline \multirow[b]{2}{*}{ Liderazgo } & Presente & Ausente \\
\hline & $\begin{array}{l}\text { Favorecimiento de acciones e } \\
\text { integración }\end{array}$ & Ausente \\
\hline Gestión de la playa & Formalizada & Sin formalidad \\
\hline
\end{tabular}

Los valores cualitativos utilizados para completar este cuadro fueron establecidos de acuerdo con el análisis comparativo de las dos localidades estudiadas. Es decir, no son valores comparables a otros casos de estudio. Ejemplo: La baja intensidad de los problemas en la playa Pocitos no necesariamente sería así definida si fuese comparada con otra playa que no fuera la Central. 
TABLA 3 - Índice de Calidad de Gobernanza (ICG) aplicado a las playas Pocitos y Central.

\begin{tabular}{lcc}
\hline Ítems del ICG & Playa Pocitos & Playa Central \\
\hline Certificación de calidad de playas & Presente & Ausente \\
\hline $\begin{array}{l}\text { Importancia que la política municipal } \\
\text { otorga a la playa }\end{array}$ & $\begin{array}{c}\text { La IDM aprueba y reconoce la política } \\
\text { ambiental del SGA, y también un plan urbano } \\
\text { formulado para la ciudad, ambos definiendo } \\
\text { objetivos para la zona costera }\end{array}$ & $\begin{array}{c}\text { El plan urbano municipal destaca la necesidad } \\
\text { de manten calidad del agua de las playas y } \\
\text { el aceno a ellas, y la preservación de } \\
\text { la arena le vegetación de dunas }\end{array}$ \\
\hline
\end{tabular}

\begin{abstract}
Período que abarca el plan estratégico
planeamiento de acciones permite una gestión durante todo el año
\end{abstract}

Existen objetivos planteados y se promueve su consecución

No hay ningún tipo de plan específico utilizado para la gestión de la playa

Existe una débil planificación de objetivos para la alta temporada, no basados en un plan específico

Ocurre durante todo el año, considerando los participantes involucrados (mayormente las divisiones de la IDM)

Coordinación activa entre los agentes implicados en la gestión de la playa

Existencia de un plan de creación de nuevos productos turísticos
Existe la idea de crear sellos de calidad para servicios asociados a las playas
Ocurre solamente entre partes del gobierno y para acciones en la alta temporada
Una de las directrices del plan de turismo inserto en $\mathrm{b}$ - es la definición de productos turísticos de acuerdo a la segmentación diversificada del mercado
Impacto del plan urbano ${ }^{\mathrm{a}, \mathrm{b}}$ para la calidad del turismo
No hay información, pero uno de los objetivos del plan urbano es planear actividades de turismo costero
No hay información, pero el plan urbano posee una sección para el turismo, en la cual el plan municipal de turismo es definido
No hay información, pero se enfatiza que Montevideo es la capital de Uruguay y de su

Nivel de cooperación con localidades adyacentes departamento correspondiente, estando dividida en municipios menores que pueden cooperar entre sí
No hay información, pero una de las directrices del plan urbano es la integración de este municipio con la region metropolitana en que se inserta

Nivel de cooperación público - privada para la gestión de playas

Ausente

Ausente

a - Plan Montevideo (2014)

b - Plano Diretor (2006)

FUENTE: Adaptado de Valls et al. (2014).

rias, destacándose la necesidad de crear y monitorear indicadores de gobernanza. Por consiguiente, se formulan las siguientes recomendaciones:

\subsubsection{Playa de Pocitos, Montevideo}

- Mejorar la institucionalidad del CP como entidad gestora de la playa, principalmente para una mejor captación de recursos y para la creación de una "dirección" a la cual los stakeholders puedan destinarse en el caso de haber interés por la temática;

- Promocionar la participación y el entendimiento general sobre las playas y el SGA, pudiéndose utilizar las más diversas herramientas existentes, tales como campañas de 
toma de conciencia sobre la importancia del sistema playa y entorno, y del rol del SGA; ampliar la divulgación de las reuniones del $\mathrm{CP}$; crear un canal de comunicación para la población; y desarrollar investigaciones de percepción de los usuarios de la playa, incorporando las respuestas a los ciclos de gestión;

- Emplear mecanismos para garantizar un proceso participativo adaptado a cada situación. Como ejemplo, la creación de una lista con el contacto de participantes actuales y potenciales para difundir invitaciones $\mathrm{y}$ actas de las reuniones y/o incorporar a dos tipos de acciones en secuencia en el CP: 1. Ampliación; cuando es necesario agregar a actores específicos para ciertos asuntos, y 2. Reducción; que corresponde a los actores tradicionales, que son responsables de llevar a cabo el plan definido, independientemente de las demandas ocurrentes en el momento (Metodología de Fuelle de Bandoneón; Poggiese, 2011);

- Crear y emplear indicadores sobre el avance del sistema y sobre el proceso de gobernanza, permitiendo identificar y apoyar las acciones de mejoría esenciales en el SGA, creando bases para un manejo adaptativo;

- Verificar la necesidad y la factibilidad de expandir la certificación ISO 14.001 a otras partes costeras de Montevideo, para lograr un gestión costera espacialmente más completa.

\subsubsection{Playa Central, Balneário Camboriú}

- Promocionar la toma de conciencia de la población y de las instituciones responsables, mejorando la comprensión general sobre las playas y de la necesidad de ser gestionadas;

- Formalizar un proceso de gestión de la playa a fin de suministrar las bases para el ordenamiento y la optimización de procedimientos, más allá de su certificación ambiental. Ésta no necesariamente debe ser por intermedio de la ISO 14.001, aunque sea la alternativa más viable hasta el momento debido a que, de entre el conjunto de certificaciones de playas existentes en todo el mundo (Williams \& Micallef, 2009), es la única que ya está formalizada en Brasil (aparte de la Bandera Azul), contando con una institución responsable por su norma (ABNT), recursos humanos capacitados para emplearla (ej. gestores e ingenieros ambientales) y empresas certificadoras experientes en su aplicación ${ }^{4}$;

- Crear una unidad para la gestión de la playa vinculada a un comité participativo, en el cual será posible integrar los stakeholders y llevar en consideración sus opiniones. Este comité puede ser integrado con algún comité ya existente donde se discuten actividades relacionadas con la temática (ej. gestión de la cuenca hidrográfica);

- Considerar la participación y el involucramiento públicos desde el principio, en todos las etapas del proceso de gestión.

\footnotetext{
4 La ISO 14.001 es una norma que establece los requisitos relativos para el empleo de un sistema de gestión ambiental en cualquier organización que se comprometa a disminuir los impactos ambientales negativos de sus actividades. de entre otros compromisos. En el caso de Brasil, fue solamente empleada en el ámbito empresarial, y no en playas.
} 


\subsubsection{Playas uruguayas y brasileñas, en general}

Finalmente, ciertos aspectos comunes fueron percibidos en ambos casos de estudio, permitiendo al establecimiento de recomendaciones generales para el proceso de gestión de playas, con la meta de contribuir a su mejora en ambos países (Figura 3):

- Gestión pública: La estructura institucional y legal de los países analizados (apartado La comprensión de la problemática) que clasifica a las playas como dominio público, confirma que su gestión debe ser de carácter público, enfatizándose que la población y los convenios público-privados son partes importantes en este proceso;

- Gestión Formalizada: Al tener en cuenta que la existencia de un acuerdo público y de un plan específico para la gestión de las playas, en el caso de Montevideo, fomentó un proceso de gestión direccionado a dichos ambientes (destacado por el análisis de la Tabla 3), se recomienda que la gestión sea formalizada por el establecimiento de leyes, acuerdos asumidos a nivel público y/o planes específicos, creando las bases para la instauración de una unidad gestora de las playas y, consecuentemente, la organización de los recursos materiales y humanos necesarios;

- Gestión Participativa: Debido a que la promoción de niveles más altos de participación demostraron beneficios a la gestión de las playas (apartado Acciones y Beneficios del Espacio de Integración), esa debe ser realizada de manera participativa con múltiples actores, a través del establecimiento de medios adecuados (ej. canal

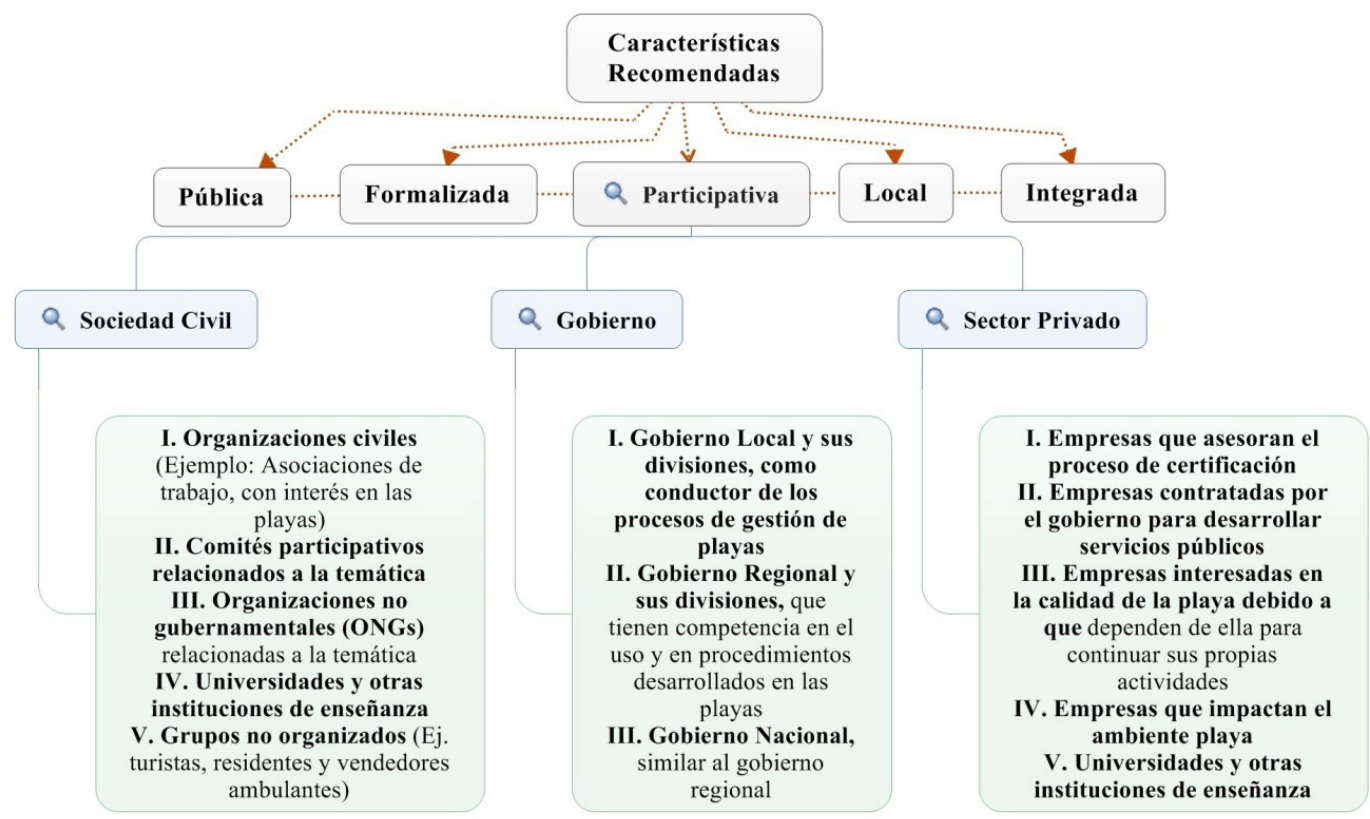

FIGURA 3 - Características generales y posibles stakeholders recomendados para la gestión de playas en Uruguay y Brasil. 
de comunicación y espacio asignados para este fin) a lo largo de todo el proceso y con la posibilidad de adaptar la intensidad y la frecuencia de la participación a cada situación y/o stakeholder. El listado de actores del presente trabajo (Tabla 1) suministró pistas sobre los participantes potenciales: 1. Gobierno (Local, Regional y Nacional), 2. Sociedad Civil (Organizaciones Civiles, Turistas, etc.), y 3. Sector Privado (Empresas contratadas por el Poder Público, Empresas interesadas en la calidad de la playa, etc.);

- Gestión Local: Al considerar que al nivel local es dónde se experimentan los problemas y los beneficios relacionados con las playas, al mismo tiempo en que los recursos y competencias para gestionarlas son limitados (ej. Ariza et al., 2008), y para garantizar el acercamiento y el monitoreo de las acciones emprendidas, se recomienda que la gestión sea conducida en la escala local. Asimismo, cabe subrayar la importancia de respetar las jerarquías y las normativas de los niveles más altos de gobierno, conservar la comunicación y el intercambio de información con ellos, y sopesar la capacidad de recursos para desarrollar la gestión;

- Gestión Integrada: Como ejemplo de la forma en la que los participantes del CP que trabajan en las divisiones de la Intendencia de Montevideo realizan un intercambio de información entre las actividades generales que desarrollan en la ciudad (gestión urbana y/o medio ambiental) y las actividades del SGA, la gestión de las playas debe ser integrada, o al menos existir una comunicación fluida con los abordajes de gestión existentes para los demás sistemas con los cuales mantiene interrelaciones: urbano, medioambiental, cuenca hidrográfica, etc. Como un caso concreto, la calidad del agua de la cuenca hidrográfica en que se ubican las playas influye directamente en la calidad del agua de estas últimas. El fin principal de esa característica es optimizar las actividades llevadas a cabo, de forma solapada, por distintos abordajes de gestión.

\section{Consideraciones finales}

Las playas urbanas son espacios dinámicos de relevancia para diversas localidades costeras, turistas y residentes. La gestión de las playas urge, por lo tanto, para minimizar los efectos negativos de diversas actividades existentes, y para maximizar los beneficios que surgen de su valor singular. En este sentido, el SGA ISO 14.001 es una alternativa que ha generado acciones beneficiosas en las playas de Montevideo.

El andamiaje institucional para la gestión de los procedimientos existentes, en Montevideo y Balneario Camboriú, se constituye principalmente por el gobierno, departamental en el primero y municipal en el segundo, siendo ausente o débil la consideración de las demás categorías de stakeholders en el proceso, en ambos casos. Asimismo, el gobierno departamental montevideano promociona la integración interna entre sus divisiones para el desarrollo del SGA, contrariamente al gobierno municipal de Balneario Camboriú, que realiza su gestión de forma sectorial y enfocada en la alta temporada. Es inminente habilitar y legitimar otros stakeholders (indicados en el presente artículo) en ambas localidades, con el objetivo de mejorar la calidad tanto de la playa como de vida de la población.

El SGA de Montevideo presenta el potencial de amparar un espacio para la gobernanza, al crear 
centralidad, liderazgo, y un ámbito de discusión, posibilitando una mejor comprensión sobre la importancia de las playas. Además, su comité participativo ha contribuido al reconocimiento de ciertas funciones de las playas, más allá de las cuestiones ambientales tradicionalmente prioridades por el SGA. La necesidad de incrementar dinámicas múltiples de participación e integración es destacada, especialmente a través de campañas de educación y empoderamiento, para cumplir con la política ambiental del SGA de las playas montevideanas. Para la gestión de la playa Central, aún muy incipiente, es fundamental la formalización de un proceso de mejora (ISO 14.001 como alternativa), a través de futuras acciones de toma de conciencia sobre la importancia de la playa.

El presente estudio encontró similitudes y diferencias entre los casos analizados, y el conjunto de recomendaciones formulado puede ser útil para la gestión de las playas de ambos países. Fue posible concluir que la gestión de las playas en Uruguay y Brasil debe ser: conducida por el poder público, si bien otros stakeholders deben ser considerados;

\section{Referencias}

AENOR - Asociación Española de Normalización y Certificación. Certificación ISO 14.001. Disponible en: <http:// www.aenor.es>. Acceso en: abril, 2014.

Albuquerque, F.; Dini, M.; Pérez, R. Nueva gestión pública, capital social y gobernanza. Sevilla: Gráficas Santa María, 2008.

Andrade, J.; Scherer, M. E. G. Decálogo da gestão costeira para Santa Catarina: avaliando a estrutura estadual para o desenvolvimento do Programa Estadual de Gerenciamento Costeiro. Desenvolvimento e Meio Ambiente, 29, 139-154, 2014. DOI: 10.5380/dma.v29ii0.31405 realizada en escala local, pero considerando las jerarquías y normas de gobierno de mayor nivel; de carácter participativo, con la integración activa de todas las partes interesadas desde el inicio del proceso; formalizada por medio de normativas locales y otros acuerdos; y, integrada a otros procesos de gestión ocurrentes en el territorio.

\section{Agradecimientos}

La investigación que da origen a los resultados presentados en la presente publicación recibió fondos de la ANII (Uruguay) bajo el código " $P O S_{-}$ $N A C \_2012$ 1_9332", por lo tanto destinamos parte de los agradecimientos a esta institución. Quisiéramos también agradecer a la CAPES (Brasil) por el soporte suministrado a través del proyecto de cooperación internacional CAPES-UdelaR “Análise Comparativa do Processo de Governança de Praias Urbanas entre Brasil e Uruguai"; y, especialmente, a todos los entrevistados y expertos consultados, sin los cuales no habría sido posible finalizar este trabajo.

Anton, S. La urbanización turística: De la conquista del viaje a la reestructuración de la ciudad turística. Documents D'Análisis Geográfica, 32, 17-43, 1998. Disponible en: $<$ http://www.redalyc.org/pdf/2818/281821983011.pdf >

Ariza, E.; Jiménez, J.; Sardá, R. A critical assessment of beach management on the Catalan coast. Ocean \& Coastal Management, 51, 141-160, 2008. doi: 10.1016/j.ocecoaman.2007.02.009

Batthyány, K.; Cabrera, M.; Alesina, L.; Bertoni, M.; Mascheroni, P.; Moreira, N.; Picasso, F.; Ramírez, J.; Rojo, V. Metodología de la investigación en Ciencias Sociales: Apuntes para un curso inicial. Montevideo: Universidad de la República, 2011. 
Bergamini, C. Uma revisão da evolução histórica dos estudos e pesquisas sobre liderança enfatiza a importância de líderes organizacionais eficazes. Revista de Administração de Empresas, 34, 3, 102-114, 1994. Disponible en: <http:// www.scielo.br/pdf/rae/v34n3/a09v34n3.pdf>.

Berkes, F.; Folke, C. (Eds.). Linking social and ecological systems. Management practices and social mechanisms for building resilience. Cambridge: Cambridge University Press, 1998.

Biasco, E. Régimen jurídico de las riberas en el Derecho uruguayo - Uso común y privativo de aguas y álveos Protección de la franja costera. Revista de la AEU, 86, 1-6, 77-121, 1999. Disponible en: <http://biblioteca3.aeu.org. uy/central/common/show_image.php?base $=$ baeu\&image $=$ / RAEU/080/086-1-77-121.pdf>.

Bombana, B.; Polette, M. Governança de praias urbanas: Adaptação do sistema de gestão ambiental de praias da UNE-EN 150.104:2008 (ISO 14001 PRAIAS) para a praia Central de Balneário Camboriú, SC - Brasil. Revista Costas, 2, 1, 1-18, 2013.

Botero, C. Utilidad de los Esquemas de Certificación de Playas para el Manejo Integrado Costero. Evaluación de ocho certificaciones en Iberoamérica. Revista Ciencia en su PC, 4, 27-41, 2009. Disponible en: <http://169.158.189.18/ cienciapc/index.php/cienciapc/article/view/72>.

Botero, C. Las Alianzas Público-privadas y Las Costas. Magazine Playascorp, 5, 2012. Disponible en: <http:// www.playascorp.com/documentos/magazine-playascorp-mayo-2012.pdf $>$.

Brasil. Lei n. 7.661, de 16 de maio de 1988. Institui o Plano Nacional de Gerenciamento Costeiro e dá outras providências. Brasília: D.O.U de 18/05/1988a.

Brasil. Constituição da República Federativa do Brasil, de 5 de outubro de 1988. 35. ed. Brasília, Centro de Documentação e Informação Edições Câmara (2012), 1988 b.

Brasil Decreto n. 5.300, de 7 de dezembro de 2004. Regulamenta a Lei n. 7.661, de 16 de maio de 1988, que institui o Plano Nacional de Gerenciamento Costeiro - PNGC. Brasília: D.O.U de 8/12/2004.

Cheong, S. M. Controlling the coast. Ocean \& Coast Management, 51, 391-396, 2008. DOI: 10.1016/j.ocecoaman.2008.01.003
Costa, H. A.; Pereira, R. M.; Hoffmann, V. E. Compreendendo o espaço turístico de Balneário Camboriú (SC) como insumo para o estudo da competitividade local turismo. Visão e Ação, 8, 2, 223-234, 2006. doi: 10.14210/rtva. v8n2.p223-234

Defeo, O.; McLachlan, A.; Schoeman, D. S.; Schlacher, T. A.; Dugan, J.; Jones, A.; Lastra, M.; Scapini, F. Threats to sandy beach ecosystems: A review. Estuarine, Coastal and Shelf Science, 81(1), 1-12, 2009. doi: 10.1016/j. ecss.2008.09.022

Ecoplata. Montevideo, 2012. Disponible en <http://taat. fcien.edu.uy/Ecoplata $/ \mathrm{html} / \mathrm{montevideo.html}>$. Acceso en: ago, 2012.

Ehler, C. Indicators to measure governance performance in integrated coastal management. Ocean \& Coastal Management, 46, 335-345, 2003. doi: 10.1016/S09645691(03)00020-6

Frey, K. Abordagens de governança em áreas metropolitanas da América Latina: avanços e entraves. Urbe. Revista Brasileira de Gestão Urbana, 4, 1, 87-102, 2012. doi: 10.1590/S2175-33692012000100007

Gutiérrez, F. Naturaleza jurídica de los municipios. Revista de Derecho Público, 22, 43, 69-73, 2013. Disponible en: $<$ http://www.revistaderechopublico.com.uy/archivos/43/ Fulvio.pdf $>$.

Gutiérrez, O. Dinámica Sedimentaria en la Costa Uruguaya: Evolución y Tendencias de Playas Urbanas en el Marco del Cambio Global. Montevideo (Uruguay), Tesis (Maestría en Geociencias) - Facultad de Ciencias, UdelaR, 2010. Disponible en: $<$ http://ambiente.fcien.edu.uy/tesis/ TesisOfeliaGutierrez.pdf $>$.

IBGE - Instituto Brasileiro de Geografia e Estatística. IBGE CIDADES@, 2011. Disponible en: <http://www.ibge.gov. br/cidadesat/topwindow.htm?1>. Acceso en: ago de 2012.

IDM - Intendencia Departamental de Montevideo. Política Ambiental: Resolución 979/12, 2012. Montevideo (Uruguay).

IDM - Intendencia Departamental de Montevideo. Playas, 2013a. Disponible en: <http://www.montevideo.gub.uy/ciudadania/desarrollo-ambiental/playas/gestion-ambiental $>$. Acceso en: ago, 2012. 
IDM - Intendencia Departamental de Montevideo. Pocitos, 2013b. Disponible en: <http://www.montevideo.gub.uy/ ciudad/historia/barrios/pocitos $>$. Acceso en: ago, 2012.

INE - Instituto Nacional de Estadística. Censos 2011: Contame que te cuento, 2011. Disponible en: $<$ http://www.ine. gub.uy/web/guest/censos-2011>. Acceso en: ago de 2012.

IPCC - Intergovernmental Pannel for Climate Change. Climate Change 2014: Impacts, Adaptation and Vulnerability, 2014. Disponible en: <http://www.ipcc.ch/report/ ar5/wg2/>. Acceso en: ago de 2014.

Ittekkot, V. Oceans, seas and sustainable development: Preparedness of developing countries. Environmental Development, 13, 46-49, 2015. doi: 10.1016/j.envdev.2014.12.001

Marchese, L.; Polette, M.; Conde, D. La gobernanza en el funcionamiento de los servicios del Sistema de Gestión Ambiental de Playas de Montevideo (Uruguay). Sustentabilidade em Debate, 4, 2, 62-82, 2013. doi: 10.18472\%2FSustDeb.v4n2.2013.9487

MEC - Ministerio de Educación y Cultura. Bienes protegidos, 2011. Disponible en: <http://www.patrimoniouruguay. gub.uy/innovaportal/v/33442/35/mecweb/patrimonio_mat erial 3 colid $=33440$ andbreadid $=$ null $>$. Acceso en: marzo, 2014.

Micallef, A.; Williams, A. T. Theoretical strategy considerations for beach management. Ocean and Coastal Management, 45, 4-5, 261-275, 2002. doi: 10.1016/S09645691(02)00058-3

MinTur - Ministério do Turismo. Turismo de Sol e Praia: Orientações Básicas, 2010. Disponible en: <http://www. turismo.gov.br/export/sites/default/turismo/o_ministerio/ acoes/downloads_publicacoes/Turismo_de_Sol_e_Praia_ Versxo_Final_IMPRESSxO_.pdf>. Acceso en: ene, 2014.

MTD - Ministerio de Turismo y Deporte. Montevideo, 2014. Disponible en: $<$ https://www.turismo.gub.uy/index.php/es/ montevideo/pocitos $>$. Acceso en: ene, 2014.

Oliveira, M. R. L; Nicolodi, J. L. A gestão costeira no Brasil e os dez anos do Projeto Orla. Uma análise sob a ótica do poder público. Revista da Gestão Costeira Integrada, 12(1), 89-98, 2012. doi: 10.5894/rgci308.

Olsen, S.; Ochoa, E. El porqué y el cómo de una línea de base para gobernanza en los ecosistemas costeros. Ecocos- tas. Guayaquil (Ecuador), noviembre, 2007. Disponible en: $<$ http://pdf.usaid.gov/pdf_docs/pnaeb386.pdf $>$.

PMBC - Prefeitura Municipal de Balneário Camboriú. Arquivo Histórico. Disponible en: $<$ http://www.balneariocamboriu.sc.gov.br/arquivo/?toda=1>. Acceso en: ene, 2013.

Poggiese, H. Consejos participativos de políticas públicas ("fuelle de bandoneón"). In: Planificación Participativa y Gestión Asociada (PPGA): Metodologías. Buenos Aires: Espacio Editorial, 2011.

Poitras, J.; Bowen, R.; Wiggin, J. 2003. Challenges to the use of consensus building in integrated coastal management. Ocean \& Coastal Management, 46, 391-405. doi: 10.1016/ S0964-5691(03)00021-8

Pomeroy, R.; Douvere, F. The engagement of stakeholders in the marine spatial planning process. Marine Policy, 32, 816-822, 2008. doi: 10.1016/j.marpol.2008.03.017

Scherer, M. Gestão de praias no Brasil: Subsídios para uma reflexão. Journal of Integrated Coastal Zone Management, 13(1), 3-13, 2013. doi: 10.5894/rgci358

Shah, A.; Treby, E.; May, V.; Walsh, P. Bridging the divide between academia and practioners: Training coastal zone managers. Ocean \& Coastal Management, 50, 859-871, 2007. doi: 10.1016/j.ocecoaman.2007.05.002

Skalee, M.; Reis, A. F. Crescimento urbano-turístico: traçado e permanências urbanas em Balneário Camboriú. In: Actas del X Coloquio Internacional de Geocrítica. Barcelona, 26-30 de mayo, 2008. Disponible en: $<$ http://www. ub.es/geocrit/-xcol/184.htm>.

Uruguay. Ley n. 18.308, de 18 de junio de 2008. Ordenamiento Territorial y Desarrollo Sostenible. Montevideo: D.O de 30/06/2008.

Uruguay. Ley n. 18.567, de 13 de setiembre de 2009. Ley de Descentralización Política y Participación Ciudadana. Montevideo: D.O de 19/10/2009.

Valls, J. F.; Rucabado, J.; Sardá, R. La playa como elemento estratégico de la gobernanza de las poblaciones de costa españolas. In: Sardá, R.; Pintó, J.; Valls, J. (Orgs.). Hacia un nuevo modelo integral de gestión de playas. Cataluña (España): Documenta Universitaria, 2014. p. 139-166.

Vaz, B.; Williams, A. T.; Pereira da Silva, C.; Phillips, M. The importance of users perception for beach management. Journal of Coastal Research, 56, 1164-1168, 2009. 
Veneziano, A. La participación ciudadana en la descentralización de Montevideo: aprendizajes y reflexiones desde los noventa. Revista Uruguaya de Ciencia Política, 17, 1, 203-227, 2008. Disponible en: <http://www.redalyc.org/ pdf/2973/297322673009.pdf $>$.

Whittingham, M. V. Aportes de la teoría y la praxis para la nueva gobernanza. Revista del CLAD Reforma y Democracia, 33, 2005. Disponible en: $<\mathrm{http} / / /$ siare.clad.org/ revistas/0043406.pdf>.

Williams, A.; Micallef, A. Beach Management: Principles and Practice. Londres: Earthscan, 2009.
Wright, R.; Stein, M. Snowball sampling. In: KemptoLeonard, K. (Org.). Encyclopedia of Social Measurement. Texas: Academic Press, 2005. p. 495-500.

Yepes, V. Gestión del uso y explotación de las playas. Cuadernos de Turismo, 19, 241-254, 2007. Disponible en: $<$ http://revistas.um.es/turismo/article/view/13731/13261>.

Yepes, V. Sistemas de gestión de calidad y medio ambiente como soporte de la gestión municipal de las playas. Equipamiento y Servicios Municipales, 117, 52-62, 2005.

Zielinski, S.; Botero, C. Guía básica para certificación de playas turísticas. Santa Marta (Colombia): Editorial Gente Nueva, 2012. $86 \mathrm{p}$. 